\title{
Monitoring suPAR levels in post-kidney transplant focal segmental glomerulosclerosis treated with therapeutic plasma exchange and rituximab
}

\author{
Nada Alachkar ${ }^{1,4^{*}}$ D, Jing Li², Dany Matar ${ }^{1}$, Vikas Vujjini ${ }^{3}$, Sami Alasfar ${ }^{1}$, Melissa Tracy $^{2}$, Jochen Reiser ${ }^{2}$ and
} Changli Wei ${ }^{2^{*}}$

\begin{abstract}
Background: Therapeutic plasma exchange (TPE) is an important therapy for recurrent focal segmental glomerulosclerosis (rFSGS) post kidney transplant. suPAR has been causally implicated in rFSGS, and shown to be a unique biomarker for the occurrence and progression of chronic kidney disease. This study was targeted to evaluate the application of monitoring suPAR in TPE treated rFSGS.

Methods: A retrospective $(n=19)$ and a prospective $(n=15)$ cohort of post transplant FSGS patients treated with TPE and rituximab were enrolled. We measured serum suPAR levels before and after the combined therapies, and assessed the role of suPAR changes on proteinuria reduction and podocyte $\beta 3$ - integrin activity.

Results: Treatment with TPE and rituximab resulted in significant decrease in proteinuria and suPAR levels. Among the variables including baseline suPAR, serum creatinine, proteinuria, eGFR, age at diagnosis, age at transplantation, transplantation numbers, time to recurrence, and TPE course numbers, only the reduction in suPAR levels and baseline proteinuria significantly correlated with the changes in proteinuria after treatment, with the former performed better in predicting proteinuria alteration. Additionally, the mean podocyte $\beta 3$ integrin activity significantly decreased after TPE and rituximab treatment $(1.10 \pm 0.08)$ as compared to before treatment $(1.34 \pm 0.08), p<0.05$. Only the reduction in suPAR predicted the response to therapies with an odds ratio of $1.43,95 \% \mathrm{Cl}(1.02,2.00), p<0.05$.

Conclusions: Serum sUPAR levels reduced significantly after TPE and rituximab treatment in post transplant FSGS patients. The reduction in suPAR levels may be utilized to assess the changes in proteinuria and monitor the response to the therapies. Larger, multi-centered prospective studies monitoring serum suPAR levels in TPE managed post transplant FSGS are warranted.
\end{abstract}

Keywords: Kidney transplant, FSGS, suPAR, Therapeutic plasma exchange

\section{Background}

Focal segmental glomerulosclerosis (FSGS) is a progressive renal disease with high probability for recurrence after renal transplantation [1,2]. Therapeutic plasma exchange (TPE) is an extracorporeal blood purification technique designed for the removal of large molecular

\footnotetext{
*Correspondence: nalachk1@jhmi.edu; changli_wei@rush.edu

'Department of Medicine, Division of Nephrology, The Johns Hopkins

University School of Medicine, Baltimore, USA

${ }^{2}$ Department of Medicine, Rush University Medical Center, 1735 W Harrison

ST, Cohn Bldg, 7th Floor, Suite 716, Chicago, IL 60612, USA

Full list of author information is available at the end of the article
}

weight substances from the plasma. Since the first report in 1985 [3], TPE has become an important therapy with variable success in recurrent FSGS (rFSGS) $[4,5]$. The application of TPE was originated from the concept of the disease causative circulating factor(s) in rFSGS [6].

Insights into podocyte biology have identified plasminogen activator, urokinase receptor (UPAR) as an important component in the maintenance of a functioning podocyte foot process structure that is regulated by lipid-dependent activation of $\alpha v \beta 3$ integrin [7]. UPAR is a glycosyl-phosphatidylinisotol (GPI)-anchored protein,

(C) The Author(s). 2018 Open Access This article is distributed under the terms of the Creative Commons Attribution 4.0 International License (http://creativecommons.org/licenses/by/4.0/), which permits unrestricted use, distribution, and 
which can be released from the plasma membrane as a soluble molecule (suPAR) by cleavage of the GPI anchor or secreted directly from cells as an alternative transcript [8]. We have identified suPAR as a circulating factor implicated in the majority of FSGS cases $[9,10]$. Yet, the relevance of suPAR to kidney injury has been surprisingly broadened recently. Several longitudinal studies in a variety of patient cohorts (cardiac risk, healthy middle-aged, pre-diabetic, dialysis patients) have found that baseline circulating suPAR levels predict chronic kidney disease (CKD) incidence and progression [11-13], strongly suggesting the application of circulating suPAR as a biomarker for monitoring CKD.

As TPE has been found to remove suPAR from blood circulation in several studies $[9,14-16]$, we sought to evaluate the application of serum suPAR as a biomarker monitoring TPE treated rFSGS patients in this study.

\section{Methods Study cohorts}

We conducted both retrospective and prospective studies of patients with FSGS as the cause of end stage renal disease (ESRD). Only patients who received TPE alone or together with rituximab for recurrent FSGS treatment were enrolled. All subjects received immunosuppressive therapy consisted of induction with thymoglobulin (1.5 $\mathrm{mg} / \mathrm{kg} /$ day) and steroid ( $500 \mathrm{mg} /$ day), followed by maintenance treatment with tacrolimus, mycophenolate mofetil and prednisone. Both studies were approved by the institutional review board (IRB) of Johns Hopkins Hospital. In the retrospective study cohort, we included 19 adult renal transplant recipients who underwent renal transplantation between September 1, 2008 to December 31, 2011 in our center and developed rFSGS after kidney transplantation. For the purpose of our prospective study, we enrolled 15 patients who were transplanted in our center between August 1, 2011 and May 31, 2015 and developed rFSGS post transplant. We followed these participants as per our research protocol and collected serum, and plasma waste samples before and after each TPE. We also evaluated the subjects' response to TPE treatment. Response to therapy is defined as reduction of proteinuria to less than $1 \mathrm{~g} / \mathrm{g}$, or the reduction of proteinuria by more than $50 \%$. The demographic and clinical characteristics of both the retrospective and the prospective cohorts are shown in Table 1.

The diagnosis of recurrent and de novo FSGS was made by the new onset of proteinuria as measured by urine protein-creatinine ratio (UPCR) of more than $1 \mathrm{~g} / \mathrm{g}$ and confirmed by kidney biopsy. Kidney biopsy in early stages showed only significant podocyte effacement on electron microscopy. Classical findings of light microscopic changes are also seen.

\section{suPAR measurement}

The measurement of serum suPAR was performed using a Human uPAR Quantikine ELISA kit (R\&D Systems Inc) following the manufacturer's instruction $[9,10]$. Standards were run three times to calculate the intra-assay coefficient of variation $(\mathrm{CV})$. The mean and SD for standard 1, standard 2, and so forth were used to derive the $\mathrm{CV}$ before averaging the $\mathrm{CV}$ of each standard. The inter-assay $\mathrm{CV}$ was derived by calculating the mean and SD for standard 1 (e.g., measurement day 1 and day 2), standard 2 (day 1 and day 2), and so forth to derive the $\mathrm{CV}$ and then average the $\mathrm{CV}$. Both the intra-assay and inter-assay $\mathrm{CV}$ s were $<5 \%$ for suPAR.

\section{Induced podocyte $\beta 3$ integrin activity assay}

To semi-quantitatively examine the effect of FSGS patient sera on podocyte $\beta 3$ integrin activity, a human podocyte cell line was cultured at $37^{\circ} \mathrm{C}$ for 14 days for complete differentiation [17]. The cells were then incubated in 5\% of FSGS patient serum for $24 \mathrm{~h}$ with lipopolysaccharide (LPS) as a positive control. Next, the cells were fixed with $4 \%$ paraformaldehyde (PFA) and processed for immunofluorescence staining for AP5 (Blood Center of Wisconsin) and paxillin (Millipore). AP5 is an antibody detecting the active state of $\beta 3$ integrin by recognizing the unfolding $\mathrm{N}$-terminal epitope GPNICT upon the activation of the integrin [18]. After immunostaining, confocal (Leica) images were taken to quantify the AP5 and paxillin intensity for each sample treatment. Paxillin signal was used to correct AP5 signal for each treatment. The relative AP5 signal (AP5/paxillin ratio) from each patient serum was then normalized against that of normal blood donor included in each assay for final report [15]. To control for suPAR specificity, the cells were co-incubated with both FSGS sera and suPAR blocking antibody. The normalized AP5 value from normal serum treated podocytes was 1 . The relative AP5 value of 1.05 or more obtained from patient serum treated podocytes was considered abnormal.

\section{Statistical analysis}

For continuous variables, data are expressed as mean \pm SEM or median with interquartile range as appropriate. Categorical variables were expressed as percentages. The demographic and clinical characteristics of patient and control participants were compared using the $t$ test, or the Fisher's exact test for categorical variables. Multiple linear or logistic regression analyses were performed to evaluate the association between serum suPAR and the variables of interest while controlling for age, sex, and other potential confounders with SPSS software. The relative change in suPAR after TPE treatment was calculated as per $10 \%$ reduction from before TPE treatment. The relative change of proteinuria in terms of UPCR 
Table 1 Demographic and Clinical Characteristics of Participants

\begin{tabular}{|c|c|c|c|}
\hline & $\begin{array}{l}\text { Retrospective } \\
(n=19)\end{array}$ & $\begin{array}{l}\text { Prospective } \\
(n=15)\end{array}$ & $P$ value \\
\hline Male, n (\%) & $8(42)$ & $10(67)$ & 0.185 \\
\hline Black, n (\%) & $11(58)$ & $5(33)$ & 0.185 \\
\hline Mean Age at Tx, yr. \pm SD & $40 \pm 12$ & $38 \pm 16$ & 0.703 \\
\hline Mean Age at native FSGS Diagnosis, yr. \pm SD & $29 \pm 8$ & $30 \pm 17$ & 0.859 \\
\hline Median Duration on Dialysis, yr. (IQR) & $2.5(1.5,8.0)$ & $3.8(1.3,6.0)$ & 0.888 \\
\hline Pre-Tx Urine, n (\%) & $13(68)$ & $6(40)$ & 0.165 \\
\hline Mean Pre-Transplant Proteinuria, g/g $\pm S D$ & $9.43 \pm 12$ & $8.88 \pm 7.32$ & 0.673 \\
\hline \multicolumn{4}{|l|}{ Primary Pre-Tx Diagnosis, n (\%) } \\
\hline FSGS & $17(89)$ & $15(100)$ & 0.492 \\
\hline Other* & $2(11)$ & & \\
\hline \multicolumn{4}{|l|}{ No. of Transplants, n (\%) } \\
\hline 1 & $12(63)$ & $8(53)$ & \multirow[t]{3}{*}{0.659} \\
\hline 2 & $4(21)$ & $5(33)$ & \\
\hline 3 & $3(16)$ & $1(7)$ & \\
\hline Living Donor, n (\%) & $12(63)$ & $11(73)$ & 0.469 \\
\hline Related & $5(26)$ & $3(20)$ & \\
\hline Unrelated & $7(37)$ & $8(53)$ & \\
\hline ABO-Incompatible Tx, n (\%) & $4(21)$ & $1(7)$ & 0.633 \\
\hline Median Time to Post-Tx FSGS, days (IQR) & $31(5,238)$ & $33(5,299)$ & 0.589 \\
\hline Mean Proteinuria at time of Post-Tx FSGS, g/g \pm SD & $4.7 \pm 3.6$ & $3.2 \pm 2.8$ & 0.366 \\
\hline Mean Peak Proteinuria, $\mathrm{g} / \mathrm{g} \pm \mathrm{SD}$ & $10.94 \pm 11.61$ & $4.6 \pm 3.25$ & 0.061 \\
\hline Median Serum Creatinine at time of Post-Tx FSGS, mg/dL (IQR) & $2.5(1.7,3.5)$ & $1.5(1.3,2.4)$ & 0.310 \\
\hline Median eGFR at time of Post-Txp FSGS, mg/dL (IQR) & $32(14,44)$ & $55(29,61)$ & 0.943 \\
\hline Median TPE (IQR) & $14(10,27)$ & $10(10,19)$ & 0.447 \\
\hline
\end{tabular}

yr year, $S D$ standard deviation, IQR interquartile range, $g$ gram, $T x$ transplant

was calculated as $100 \times$ (UPCR before treatment-UPCR after treatment)/UPCR before treatment. All statistical tests were two tailed. $P$ values $<0.05$ were considered significant.

\section{Results}

\section{Single course of TPE on sUPAR removal}

To look at the immediate effect of TPE on serum suPAR levels, we compared serum suPAR right before and after a single course of TPE. We found that single course of TPE could remove on average $37 \%$ of serum suPAR (Fig. 1a). Simultaneously, suPAR was detected in the pheresis waste bags, ranging from $1149 \mathrm{pg} / \mathrm{ml}$ to 2417 $\mathrm{pg} / \mathrm{nl}$ with an average suPAR value of $1848 \mathrm{pg} / \mathrm{ml}$. This is in consistent with previous reports $[9,14-16]$, and indicates that TPE could effectively decrease serum suPAR levels by removing suPAR from the blood circulation.

\section{Historical cohort}

We performed a retrospective study, in which we analyzed 19 post-transplant (post-Tx) FSGS patients, 17 of them had rFSGS, and 2 had de novo FSGS (Table 1), $42 \%$ of them were male. The mean \pm SD age at transplant was $40 \pm 12$ years old. Median time to post-Tx FSGS (IQR) was $31(5,238)$ days. All patients were treated with TPE, and 12 received rituximab as well. Serum was collected before transplantation, after transplantation at the time of post-Tx FSGS diagnosis, and before and after TPE sessions for suPAR measurement. Each patient received a median (IQR) of TPE $14(7,82)$ sessions (Table 1). As shown in Fig. 1b, mean serum suPAR was higher before transplantation comparing to that at the time of post-Tx FSGS diagnosis $(8131 \pm 1300 \mathrm{pg} / \mathrm{ml}$ before Tx versus $5551 \pm 429.2 \mathrm{pg} / \mathrm{ml}$ post-Tx, $p=0.056$ ). One TPE session resulted in significant reduction in serum suPAR level $(5551 \pm 429 \mathrm{pg} / \mathrm{ml}$ before TPE vs $4532 \pm 351 \mathrm{pg} / \mathrm{ml}$ post TPE, $p<0.05$ ). By the end of treatment with TPE and rituximab, the mean proteinuria significantly decreased from $4.84 \pm 0.76 \mathrm{~g} / \mathrm{g}$ to $2.06 \pm 0.47$ ( $p<0.01$, Fig. $2 \mathrm{a})$. The median serum creatinine levels were significantly decreased as well $(2.5 \mathrm{mg} / \mathrm{dL}$ before therapies versus after $1.8 \mathrm{mg} / \mathrm{dL}$ after therapies, $p<0.05$, Fig. 2b). 

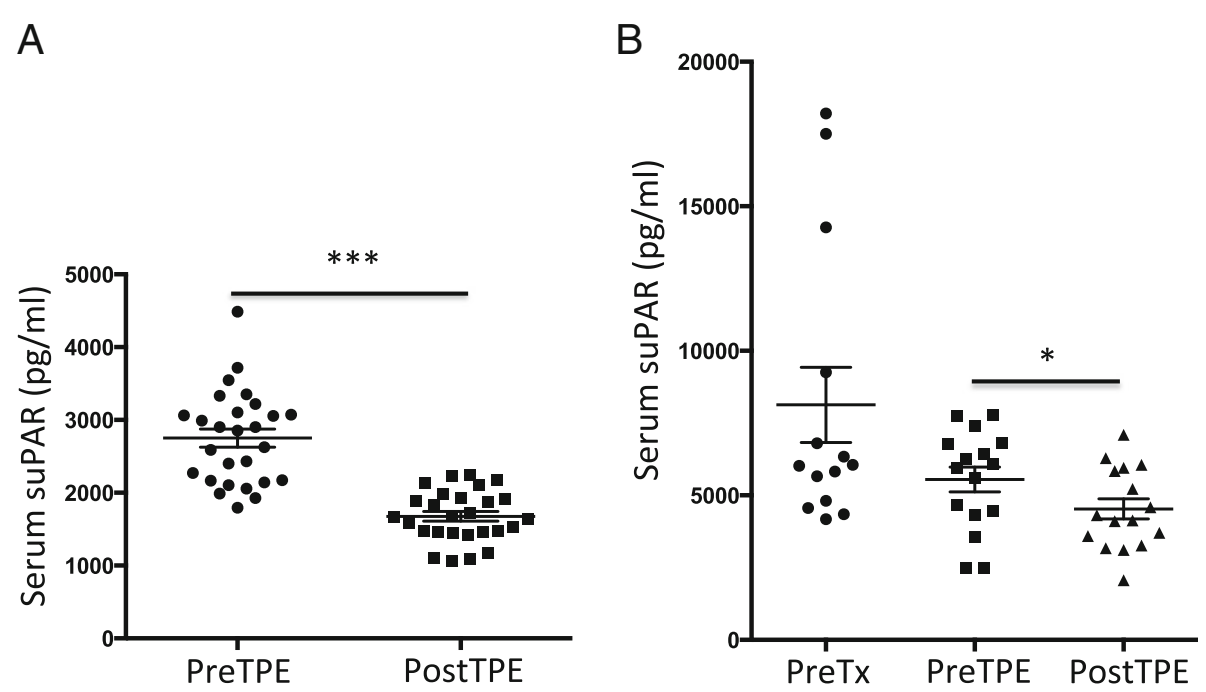

Fig. 1 TPE reduced serum sUPAR levels a Single course of TPE significantly reduced serum suPAR levels. ${ }^{* *}, p<0.001$. b TPE alone or combined therapy decreased serum suPAR levels in retrospective cohort of post transplant FSGS. * $p<0.05$

\section{Prospective cohort}

To validate our findings of the retrospective study and assess the role of suPAR in monitoring the response to TPE in post-Tx FSGS, we performed a prospective study of 15 patients with rFSGS. As indicated in Table 1, 67\% of them were male and mean \pm SD age at transplantation was $43 \pm 15$ years old. The median time to rFSGS was $33(5,299)$ days. All these patients received TPE and rituximab. The median (IQR) TPE was $10(10,52)$ sessions. Serum was collected to measure serum suPAR and creatinine levels, and urine was collected for proteinuria assessment before and after TPE treatment. We found that one TPE resulted in significant reduction in serum suPAR levels to from $3240 \pm 409.4 \mathrm{pg} / \mathrm{ml}$, to $2486 \pm 229 \mathrm{pg} / \mathrm{ml}(p<0.05$, Fig. 3a). Similarly, proteinuria decreased significantly as well $(4.19 \pm 1.14 \mathrm{~g} / \mathrm{g}$ before vs $2.07 \pm 0.75 \mathrm{~g} / \mathrm{g}$ after treatment, $p<0.01$, Fig. $3 \mathrm{~b})$. The median serum creatinine reduced but did not reach any statistical significance (Fig. 3c). Building on our previous findings that elevated circulating suPAR could induce podocyte $\beta 3$ integrin activity and thus kidney injuries [9], we performed cultured human podocyte based $\beta 3$ integrin activity assay by incubating podocytes with post-Tx FSGS patient sera harvested before and after TPE treatments (Fig. 4). The generated AP5 activity was corrected against that of normal sera from healthy subjects. Co-incubation of the patient sera with anti-human suPAR antibody was applied in order to control for the suPAR effect (Fig. 4). In consistent with the changes of serum suPAR levels and proteinuria, the mean $\beta 3$ integrin activity in terms of AP5 intensity significantly decreased after treatment $(1.10 \pm 0.08)$ as compared to before treatment $(1.34 \pm 0.08, p<0.05$, Fig. $3 \mathrm{~d})$. Before TPE
A

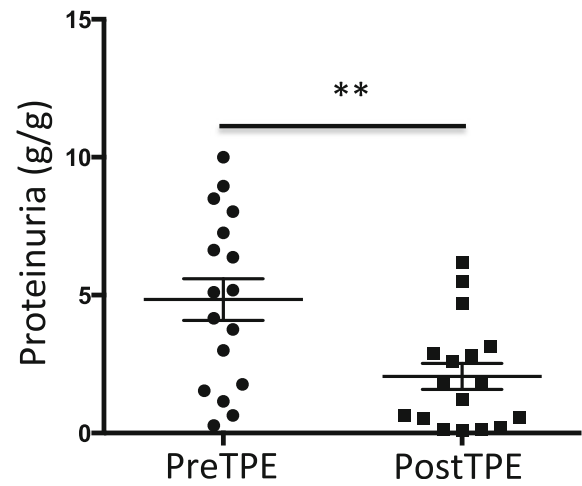

B

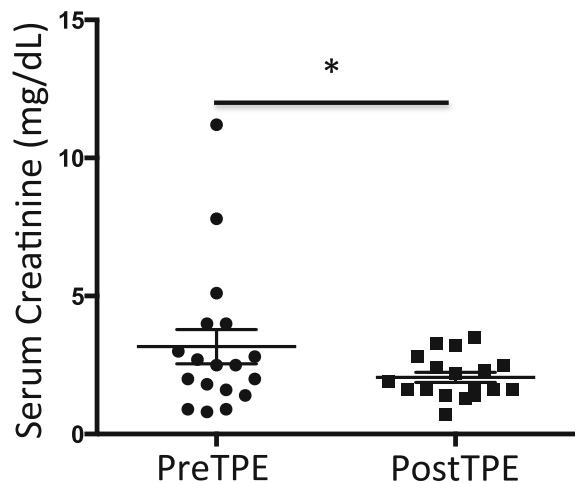

Fig. 2 Effects of TPE alone or combined therapy in retrospective cohort $\mathbf{a}$. Proteinuria was significantly decreased after TPE therapy. ${ }^{* *} p<0.01$. $\mathbf{b}$ Serum creatinine was reduced after TPE therapy. ${ }^{*} p<0.05$ 

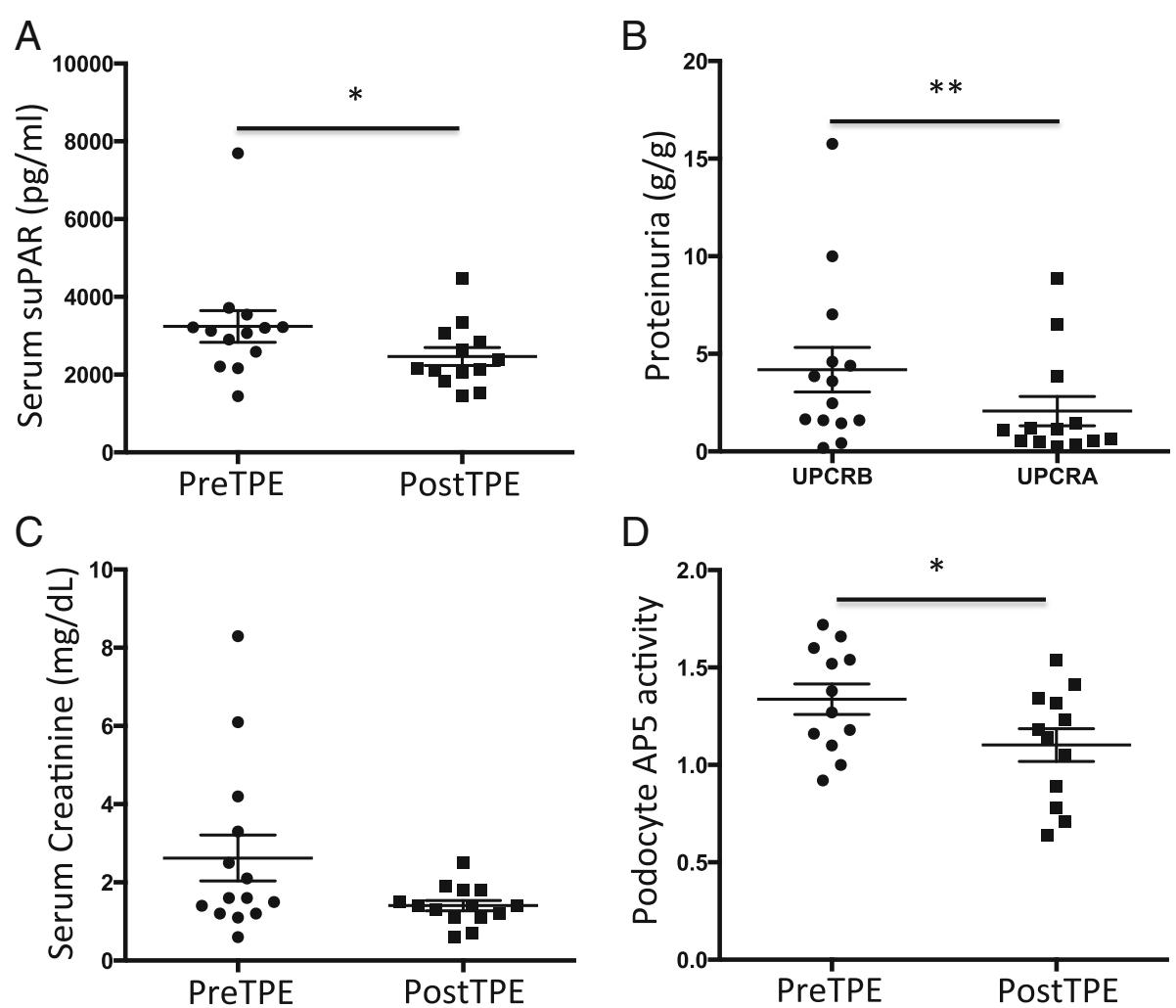

Fig. 3 Effects of TPE combined therapy in prospective cohort $\mathbf{a}$. Serum suPAR levels were significantly reduced after treatment. ${ }^{*} p<0.05 . \mathbf{b}$ Proteinuria was significantly decreased after treatment. ${ }^{* *} p<0.01$. c Serum creatinine was marginally but not significantly improved after combined therapy. $\mathbf{d}$ The patient serum induced podocyte AP5 activity was significantly reduced after treatment. ${ }^{*} p<0.05$

and rituximab combined therapies, 11 out of 12 patients had high podocyte AP5 value, while 7 of 12 patients had high AP5 after treatment. Out of 5 patients with AP5 normalized after treatment, only $1(20 \%)$ did not respond to TPE and rituximab. Among the 7 patients with persistent high podocyte AP5 values after treatment, 5 (71.4\%) did not respond to the combined therapies.

\section{Correlation of serum suPAR modification to clinical outcome}

Next, we analyzed the distribution of both retrospective and prospective cohorts and found that the demographic and clinical characteristics are similar (Table 1), thus we combined the two cohorts together for further analysis. First, we examined the bivariate correlations between the continuous variables, including serum suPAR, serum creatinine, eGFR, and UPCR before TPE treatment; age at diagnosis, age at transplantation, transplant number, TPE course numbers, as well as, the reduction in serum suPAR (dsuPAR), the reduction in UPCR (dUPCR) after treatment (Table 2). Pre-TPE levels of serum suPAR were associated with serum creatinine $(r=0.37, p<0.05)$. Pre-TPE UPCR was correlated with serum creatinine $(r=0.47, p<0.01)$, and transplant number $(r=0.38, p<0.05)$. The reduction in UPCR after treatment was correlated with Pre-TPE
UPCR $(r=0.42, p<0.05)$, and the reduction in suPAR $(r=0.48, p<0.01)$.

Then, we performed multiple linear regression analysis to evaluate the reduction in UPCR after treatment, controlling for Pre-TPE suPAR, UPCR, serum creatinine, eGFR, and patient gender, TPE number, age at transplant, transplant number, as well as the reduction in suPAR post-TPE treatment. We observed that the reduction in serum suPAR levels alone accounted for $23 \%$ proteinuria reduction. The reduction in serum suPAR levels and baseline UPCR together accounted for $35 \%$ proteinuria reduction after treatment, with the reduction in serum suPAR still predicting stronger than baseline UPCR (Table 3). Lastly, we performed a logistic multiple regression to look at the response to TPE and rituximab combined therapies, which was defined as UPCR $<1 \mathrm{~g} / \mathrm{g}$ and/or the reduction in UPCR more than $50 \%$, controlling for variables including the reduction in serum suPAR levels, Pre-TPE UPCR, eGFR, TPE number and age at transplant. We found that only the reduction in serum suPAR significantly predicts the response to the therapies with an odds ratio of $1.43,95 \%$ CI $(1.02,2.00), p<0.05$.

\section{Discussion}

Originating from the concept of circulating permeability factor(s), TPE has become an important therapy in 


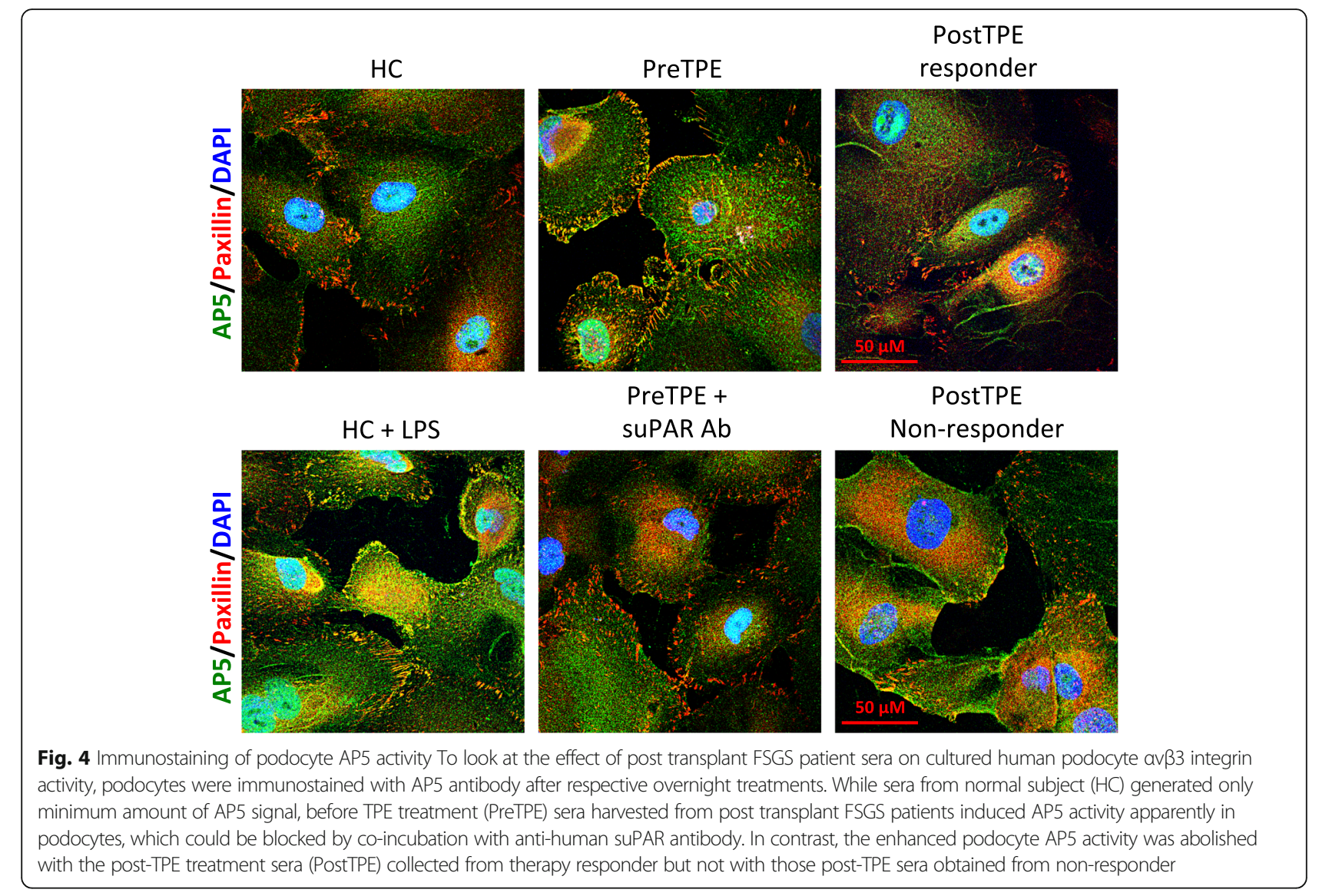

Table 2 Correlations between different variables

\begin{tabular}{|l|l|l|l|l|l|l|l|l|l|l|l|}
\hline & suPAR & UPCR & SCR & TPEs & Tx age & Txs & dsuPAR & Dx age & $\begin{array}{l}\text { Time to } \\
\text { recur }\end{array}$ & dUPCR & eGFR \\
\hline suPAR & & 0.34 & $0.37^{*}$ & 0.31 & 0.16 & -0.21 & $0.47^{*}$ & 0.31 & 0.09 & 0.26 & -0.11 \\
\hline UPCR & 0.067 & & $0.47^{* *}$ & 0.04 & 0.16 & $0.38^{*}$ & 0.25 & 0.20 & 0.04 & $0.42^{*}$ & -0.19 \\
\hline SCR & 0.045 & 0.007 & & 0.005 & -0.19 & -0.31 & 0.30 & 0.02 & 0.002 & 0.22 & $0.53^{* *}$ \\
\hline TPEs & 0.099 & 0.82 & 0.98 & & 0.1 & 0 & 0.07 & -0.014 & -0.24 & -0.21 & -0.05 \\
\hline Tx age & 0.40 & 0.39 & 0.32 & 0.59 & & -0.15 & -0.24 & $0.77^{* *}$ & -0.13 & -0.16 & 0.05 \\
\hline Txs & 0.29 & 0.035 & 0.094 & 0.998 & 0.42 & & 0.09 & -0.18 & 0.05 & 0.08 & 0.22 \\
\hline dsuPAR & 0.012 & 0.20 & 0.13 & 0.73 & 0.23 & 0.64 & & 0.06 & -0.014 & $0.48^{* *}$ & 0.11 \\
\hline Dx age & 0.13 & 0.33 & 0.93 & 0.95 & 0.000 & 0.37 & 0.78 & & -0.15 & -0.05 & -0.04 \\
\hline Time to & 0.65 & 0.84 & 0.99 & 0.19 & 0.50 & 0.78 & 0.95 & 0.46 & & 0.07 & -0.22 \\
Recur & & & & & & & & & & & \\
\hline dUPCR & 0.18 & 0.018 & 0.22 & 0.25 & 0.38 & 0.66 & 0.01 & 0.81 & 0.72 & & 0.14 \\
\hline eGFR & 0.59 & 0.31 & 0.002 & 0.79 & 0.81 & 0.23 & 0.58 & 0.83 & 0.24 & 0.45 & \\
\hline
\end{tabular}

Upper in black, Pearson $r$ vlue; lower in blue, $p$ value. dUPCR, the relative change in UPCR; dsuPAR, the relative change in serum suPAR levels; UPCR, before TPE urinary protein creatinine ratio; SCR, before TPE serum creatinine; Tx age, age at transplant; Tx\#, transplant times; TPE\#, the number of TPE courses treated; Dx age, age at diagnosis; $\mathrm{dUPCR}=100 \times$ (UPCR PreTPE-UPCR POSTTPE)/UPCR PreTPE;

dsuPAR $=$ Per $10 \%$ reduction from PreTPE levels of suPAR

*Correlation is significant at the 0.05 level (2-tailed); ** Correlation is significant at the 0.01 level (2-tailed) 
Table 3 Multiple linear regression analysis of proteinuria change

\begin{tabular}{llllll}
\hline Model & B & SEM & $\beta$ & $P$ value \\
\hline 1 & Constant & 20.84 & 13.22 & & \\
& Relative change in SUPAR & 9.84 & 3.54 & 0.48 & 0.01 \\
2 & Constant & -11.3 & 19.56 & & \\
& Relative change in sUPAR & 8.00 & 3.43 & 0.39 & 0.03 \\
& Baseline UPCR & 7.08 & 3.33 & 0.36 & 0.04 \\
\hline UPCR urine protein-creatinine ratio & & & &
\end{tabular}

recurrent FSGS patients. A recent systematic review of patients with rFSGS indicates that $71 \%$ of patients achieved full or partial remission after treatment with TPE [19]. Yet, there has been no biomarker available to gauge TPE therapy, largely due to the delay in identifying the responsible circulating factor(s). In this study containing both retrospective and prospective rFSGS cohorts, we found that serum suPAR was reduced after TPE treatments and the reduction in suPAR could predict the response to TPE and rituximab.

While suPAR has been considered to be an inflammatory marker, implicated in many medical conditions, its pathogenic involvement in kidney has been just unfolded. Several large and diverse cohorts have shown that baseline suPAR stands out as a unique biomarker in predicting the occurrence and progression of CKD, as well as the cardiovascular events with both adult and children patients [11-13]. In terms of FSGS, we have shown that suPAR as a circulating factor can contribute to the development of FSGS, via mechanisms that activate podocyte $\alpha v \beta 3$ integrin [9]. Moreover, increased serum suPAR levels have been observed in a majority of primary FSGS patients and most rFSGS patients investigated $[9,10]$. While not all follow-up studies have reached the same conclusions [20], and indeed further multicenter large cohort studies that could adopt the same study protocols are warranted, these findings highly suggest the implication of suPAR in FSGS patients.

Recently, we have shown that the degree of podocyte effacement correlated with suPAR levels at the time of rFSGS diagnosis, and that response to therapy resulted in significant reduction of suPAR level [21]. In this study, we found that circulating suPAR could be effectively reduced by a single course of TPE. This is in consistent with other reports $[9,14-16]$, indicating that suPAR can be managed by TPE. Yet, why serum suPAR bounced back significantly in some but not other studies [15], and how to control the rebound of serum suPAR and proteinuria deserve further investigation. Of the possible explanations for the discrepancy, the difference in clinical management protocols adopted at different institutes should be accountable at least partially.

In this study of the post-Tx FSGS patients, we found that TPE reduced serum suPAR levels and suPAR-induced podocyte $\alpha v \beta 3$ integrin activity. More importantly, the decrease of serum suPAR is associated with the reduction of proteinuria. Controlling for variables including baseline serum creatinine, eGFR, proteinuria, age at transplantation, transplant numbers, and TPE course numbers, the reduction in suPAR stands out as the strongest predictor for proteinuria reduction after TPE treatments, account for $23 \%$ of proteinuria variance. In terms of response to therapy (UPCR $<1 \mathrm{~g} / \mathrm{g}$ and/or UPCR reduction $>50 \%$ ), out of many above analyzed variables, only the reduction in suPAR level can predict the outcome, indicating suPAR is an applicable biomarker in TPE managed post-Tx FSGS.

Our study has few limitations, in particular the small sample size of our cohort. Additionally, for our prospective study, we have only short-term outcome. Obviously, a multicenter prospective study that includes a larger cohort with long term follow up duration is warranted to address these issue. Nevertheless, monitoring and regulating suPAR levels in patients with rFSGS or other kidney disease deserve more attention, especially because experimental evidence is emerging that suggests inhibiting uPAR pathway by cyclo-RGDfv, uPAR antibody or most recently by UPARANT, a uPAR-derived small peptide with predominant anti-inflammatory action, could render renal protection $[7,9,22]$.

\section{Conclusions}

In this study of post transplant FSGS patients managed with TPE and rituximab, the reduction of suPAR after treatment contributed significantly to the reduction in proteinuria as well as to the response to therapy. These findings support monitoring of suPAR in TPE and rituximab managed recurrent and de novo FSGS.

\section{Abbreviations}

Cl: Confidence interval; CKD: Chronic kidney disease; CV: Coefficient of variation; eGFR: Estimated glomerular filtration ratio; ESRD: End stage renal disease; FSGS: Focal segmental glomerulosclerosis; GPI: Glycosylphosphatidylinisotol; IQR: Interquartile range; LPS: Lipopolysaccharide; PFA: Paraformaldehyde; SCR: Serum creatinine; SD: Standard deviation; SEM: Standard error of mean; suPAR: Soluble urokinase receptor; TPE: Therapeutic plasma exchange; Tx: Transplantation; UPCR: Urinary protein/creatinine ratio

\section{Acknowledgements \\ None \\ Funding \\ CW was the recipient of 2013 Plasma Exchange Innovation Award sponsored by Terumo. The funding body did not have any participation in the development of the study protocol or data collection/assessment. BCT. JR, NA and CW are supported by R01DK101350. The funding body did not have any participation in the development of the study protocol or data collection/assessment.}

Availability of data and materials

The dataset generated and analyzed during the current study is not publicly available because the interview transcripts contain personal and potentially identifying information. 


\section{Authors' contributions}

NA: data and human samples collection, analysis and manuscript writing. JL: data collection, performed experiments (suPAR measurement, cell culture and treatment, AP5 assay), data analysis. DM: clinical data and human samples collection, data analysis, manuscript writing. W: data and human samples collection, manuscript writing. SA: data collection, research design, manuscript writing. MT: research design, manuscript writing. JR: research design, manuscript writing. CW: research design, data collection and analysis, manuscript writing. All authors have read and approved the manuscript.

\section{Ethics approval and consent to participate}

This work was approved by the institutional review board (IRB) of Johns Hopkins Hospital. Written consents were obtained from the participates in the prospective study.

\section{Consent for publication}

Not applicable

\section{Competing interests}

At the time of publication, NA is a Section Editor at BMC Nephrology. CW has a pending patent on suPAR in diabetic kidney disease. JR is cofounder and stockholder of Trisaq, a biotechnological company developing novel therapeutics for chronic kidney diseases and FSGS. He has pending and issued patents in the therapeutic and diagnostic space regarding kidney diseases.

\section{Publisher's Note}

Springer Nature remains neutral with regard to jurisdictional claims in published maps and institutional affiliations.

\section{Author details}

${ }^{1}$ Department of Medicine, Division of Nephrology, The Johns Hopkins University School of Medicine, Baltimore, USA. ${ }^{2}$ Department of Medicine, Rush University Medical Center, 1735 W Harrison ST, Cohn Bldg, 7th Floor, Suite 716, Chicago, IL 60612, USA. ${ }^{3}$ Department of Medicine, Sinai Hospital, Baltimore, USA. ${ }^{4}$ Division of Nephrology, Johns Hopkins Hospital, 600 Wolfe St. Carnegie 344B, Baltimore, MD 21287, USA.

Received: 14 June 2018 Accepted: 5 December 2018

Published online: 17 December 2018

\section{References}

1. Ponticelli C, Glassock RJ. Posttransplant recurrence of primary glomerulonephritis. Clin J Am Soc Nephrol. 2010:5:2363-72. https://doi.org/ 10.2215/CJN.06720810

2. Cravedi P, Kopp JB, Remuzzi G. Recent progress in the pathophysiology and treatment of FSGS recurrence. Am J Transplant. 2014;13:266-74. https://doi. org/10.1111/ajt.12045.

3. Zimmerman SW. Plasmapheresis and dipyridamole for recurrent focal glomerular sclerosis. Nephron. 1985:40:241-5. https://doi.org/10.1159/ 000183469.

4. Clark WF, Huang SS, Walsh MW, et al. Plasampheresis for the treatment of kidney diseases. Kidney Int. 2016;90:974-84. https://doi.org/10.1016/j.kint. 2016.06.009

5. Alasfar S, Matar D, Montgomery RA, et al. Rituximab and therapeutic plasma exchange in recurrent focal segmental glomerulosclerosis post kidney transplantation. Transplantation. 2018;102:e115-20. https://doi.org/10.1097/ TP.0000000000002008

6. Fogo AB. Causes and pathogenesis of focal segmental glomerulosclerosis. Nat Rev Nephrol. 2015;11:76-87. https://doi.org/10.1038/nrneph.2014.216.

7. Wei C, Möller CC, Altintas MM, et al. Modification of kidney barrier function by the urokinase receptor. Nat Med. 2008;14:55-63. https://doi.org/10.1038/ $\mathrm{nm} 1696$.

8. Thuno M, Macho B, Eugen-Olsen J. suPAR: the molecular crystal ball. Dis Markers. 2009;27:157-72. https://doi.org/10.3233/DMA-2009-0657.

9. Wei C, El Hindi S, Li J, et al. Circulating urokinase receptor as cause for focal segmental glomerulosclerosis. Nat Med. 2011;17:952-60. https://doi.org/10. 1038/nm.2411.

10. Wei C, Trachtman H, Li J, et al. Circulating suPAR in two cohorts of primary FSGS. J Am Soc Nephrol. 2012;23:2051-9. https://doi.org/10.1681/ASN. 2012030302
11. Hayek SS, Sever S, Ko YA, et al. Soluble Urokinase receptor and chronic kidney disease. N Engl J Med. 2015;373(20):1916-25. https://doi.org/10.1056/ NEJMoa1506362.

12. Drechsler C, Hayek SS, Wei $\mathrm{C}$, et al. Soluble Urokinase plasminogen activator receptor and outcomes in patients with diabetes on hemodialysis. Clin J Am Soc Nephrol. 2017;12:1265-73. https://doi.org/10.2215/CJN.10881016.

13. Schaefer $F$, Trachtman $H$, Wühl $E$, et al. Soluble urokinase receptor and progression of kidney disease in children. JAMA Pediatr. 2017;171:e172914. https://doi.org/10.1001/jamapediatrics.2017.2914.

14. Morath C, Wei C, Macher-Goeppinger S, Schwenger V, Zeier M, Reiser J. Management of severe recurrent focal segmental glomerulosclerosis through circulating soluble urokinase receptor modification. Am J Ther. 2013;20(2):226-9. https://doi.org/10.1097/MJT.0b013e3182811aca.

15. Staeck O, Slowinski T, Lieker I, et al. Recurrent primary focal segmental glomerulosclerosis managed with intensified plasma exchange and concomitant monitoring of soluble urokinase-type plasminogen activator receptor-mediated podocyte $\beta 3$-integrin activation. Transplantation. 2015;99: 2593-7. https://doi.org/10.1097/TP.0000000000000914.

16. Schenk H, Müller-Deile J, Schmitt R, Bräsen JH, Haller H, Schiffer M. Removal of focal segmental glomerulosclerosis (FSGS) factor suPAR using CytoSorb. J Clin Apher. 2017:1-9. https://doi.org/10.1002/jca.21538.

17. Saleem MA, O'Hare MJ, Reiser J, et al. A conditionally immortalized human podocyte cell line demonstrating nephrin and podocin expression. J Am Soc Nephrol. 2002;13:630-8.

18. Honda S, Tomiyama Y, Pelletier AJ, et al. Topography of ligand-induced binding sites, including a novel cation-sensitive epitope (AP5) at the amino terminus, of the human integrin beta 3 subunit. J Biol Chem. 1995;270: 11947-54.

19. Kashgary A, Sontrop J, Li L, et al. The role of plasma exchange in treating post-transplant focal segmental glomerulosclerosis: a systematic review and meta-analysis of 77 case-reports and case-series. BMC Nephrol. 2016;17:104. https://doi.org/10.1186/s12882-016-0322-7.

20. Wada T, Nangaku M. A circulating permeability factor in focal segmental glomerulosclerosis: the hunt continues. Clin Kidney J. 2015;8:708-15. https:// doi.org/10.1093/ckj/sfv090.

21. Alachkar N, Wei C, Arend LJ, et al. Podocyte effacement closely links to suPAR levels at time of posttransplantation focal segmental glomerulosclerosis occurrence and improves with therapy. Transplantation. 2013:96(7):649-56. https://doi.org/10.1097/TP.0b013e31829eda4f.

22. Dal Monte M, Cammalleri M, Pecci V, et al. Inhibiting the urokinase-type plasminogen activator receptor system recovers STZ-induced diabetic nephropathy. J Cell Mol Med. 2018. https://doi.org/10.1111/jcmm.14004.

Ready to submit your research? Choose BMC and benefit from:

- fast, convenient online submission

- thorough peer review by experienced researchers in your field

- rapid publication on acceptance

- support for research data, including large and complex data types

- gold Open Access which fosters wider collaboration and increased citations

- maximum visibility for your research: over $100 \mathrm{M}$ website views per year

At BMC, research is always in progress.

Learn more biomedcentral.com/submissions 